\title{
Saberes e práticas de mulheres ribeirinhas no climatério: autocuidado, uso de plantas medicinais e sistemas de cuidado em saúde
}

Knowledge and practices of riverine women in the climacteric: self-care, use of medicinal plants and health care systems

Conocimientos y prácticas de las mujeres ribereñas en el climaterio: autocuidado, uso de plantas medicinales y sistemas de salud Universidade do Estado do Pará, Brasil
E-mail: oracio.junior@ @uepa.br

Adriana Olímpia Barbosa Felipe ORCID: https://orcid.org/0000-0002-4491-5750 Universidade Federal de Alfenas, Brasil E-mail: adrianaofelipe@yahoo.com.br

Cíntia Bezerra Almeida Costa

ORCID: https://orcid.org/0000-0002-1179-5852 Universidade Federal da Paraíba, Brasil E-mail: cintia.costa@academico.ufpb.br

Kaio Vinícius Paiva Albarado ORCID: https://orcid.org/0000-0002-0687-7124

Universidade Federal do Oeste do Pará, Brasil E-mail: kaioalbarado@gmail.com

Tayane Moura Martins

ORCID: https://orcid.org/0000-0003-3236-8574 Universidade Federal do Pará, Brasil

E-mail: tayane_m.martins1@ @otmail.com

Priscilla Rodrigues Caminha Carneiro ORCID: https://orcid.org/0000-0002-3385-9108 Universidade do Estado do Pará, Brasil E-mail: priscilla.caminha@uepa.br

Michele Lima de Albuquerque dos Santos ORCID: https://orcid.org/0000-0002-7273-026X Centro Universitário do Norte, Brasil E-mail: misaint.21@gmail.com

\begin{abstract}
Resumo
Objetivos: O estudo teve como objetivos compreender as práticas e saberes das mulheres ribeirinhas relativas ao climatério e autocuidado. Metodologia: Estudo descritivo exploratório, de abordagem qualitativa, realizado com as mulheres ribeirinhas residentes em comunidades localizadas ao longo da margem esquerda do Rio Amazonas. A coleta de dados se deu através de entrevistas semiestruturadas, com 24 mulheres na faixa etária entre 40 e 65 anos de idade. Utilizou-se o método de análise temática de Leininger. Resultados: Participaram do estudo vinte e quatro ribeirinhas. Vinte (20) viviam com o companheiro, doze (12) não tinham ensino médio e nove (9) eram do lar. Foram identificadas três categorias: 1) Mulheres Ribeirinhas: Percebendo-se e experenciando o climatério/menopausa 2) Mulheres Ribeirinhas e as vivencias de autocuidado e 3) Mulheres ribeirinhas experienciando os sistemas de cuidado em saúde. As mulheres possuem saberes acerca do autocuidado, passados de geração a geração, e do uso das plantas medicinais.
\end{abstract}


Considerações Finais: As mulheres ribeirinhas possuem conhecimento acerca do Climatério e lançam mão de saberes e práticas populares e do modelo biomédico para o seu autocuidado. $\mathrm{O}$ estudo reforça a importância da aproximação do saber científico com o popular, promovendo troca de saberes e experiências e consequentemente uma melhor qualidade de vida para as ribeirinhas.

Palavras-chave: Enfermagem Transcultural; Saúde da mulher, Climatério, Autocuidado, População rural, Plantas Medicinais.

\begin{abstract}
Objectives: The study aimed to understand the practices and knowledge of riverside women regarding climacteric and self-care. Methodology: Descriptive exploratory study, with a qualitative approach, carried out with riverine women living in communities located along the left bank of the Amazon River. Data collection took place through semistructured interviews with 24 women aged between 40 and 65 years old. Leininger's thematic analysis method was used. Results: Twenty-four riverside dwellers participated in the study. Twenty (20) lived with a partner, twelve (12) did not have high school and nine (9) were housewives. Three categories were identified: 1) Riverside women: Perceiving and experiencing the climacteric/menopause 2) Riverside women and self-care experiences and 3) Riverside women experiencing health care systems. Women have knowledge about self-care, passed from generation to generation, and the use of medicinal plants. Final Considerations: Riverside women have knowledge about Climacteric and make use of popular knowledge and practices and the biomedical model for their self-care. The study reinforces the importance of bringing scientific and popular knowledge closer together, promoting the exchange of knowledge and experiences and, consequently, a better quality of life for riverside dwellers.
\end{abstract}

Keywords: Transcultural Nursing; Women's health, Climacteric, Self-care, Rural population, Medicinal plants.

\title{
Resumen
}

Objetivos: El estudio tuvo como objetivo comprender las prácticas y conocimientos de las mujeres ribereñas en materia de climaterio y autocuidado. Metodología: Estudio exploratorio descriptivo, con enfoque cualitativo, realizado con mujeres ribereñas residentes en comunidades ubicadas a lo largo de la margen izquierda del río Amazonas. La recolección de datos se realizó mediante entrevistas semiestructuradas a 24 mujeres de entre 40 y 65 años. Se utilizó el método de análisis temático de Leininger. Resultados: Participaron en el estudio veinticuatro habitantes ribereños. Veinte (20) vivían en pareja, doce (12) no tenían bachillerato y nueve (9) eran amas de casa. Se identificaron tres categorías: 1) Mujeres de Riverside: Percibiendo y experimentando el climaterio / menopausia 2) Mujeres de Riverside y experiencias de autocuidado y 3) Mujeres de Riverside experimentando sistemas de salud. Las mujeres tienen conocimientos sobre el autocuidado, transmitidos de generación en generación, y el uso de plantas medicinales. Consideraciones finales: Las mujeres de Riverside tienen conocimientos sobre el climaterio y utilizan los conocimientos y prácticas populares y el modelo biomédico para su autocuidado. El estudio refuerza la importancia de acercar el conocimiento científico y popular, promoviendo el intercambio de conocimientos y experiencias y, en consecuencia, una mejor calidad de vida de los ribereños.

Palabras clave: Enfermería Transcultural; Salud de la mujer, Climaterio, Autocuidado, Población rural, Plantas medicinales.

\section{Introdução}

As mulheres constituem a grande maioria da população brasileira, sendo consideradas as principais usuárias do Sistema Único de Saúde (SUS). De acordo com dados da PNAD Contínua (Pesquisa Nacional por Amostra de Domicílios Contínua) 2019, o número de mulheres no Brasil é superior ao de homens, sendo a população composta por 48,2\% de homens e 51,8\% de mulheres (IBGE, 2019). Dentro desse universo, milhões de mulheres se encontram no período identificado pelo Ministério como climatério com início por volta dos 40 anos e se estendendo até os 65 anos, logo, em torno de $34 \%$ das mulheres brasileiras estão nesta fase definida como o período compreendido pela transição entre a fase reprodutiva e nãoreprodutiva da vida da mulher (Brasil, 2008).

Durante o Climatério, modificações nos ovários diminuem a produção de estrogênio se tornando insuficiente para garantir a reprodução e a manutenção das características funcionais dos órgãos sexuais femininos. Esse declínio faz com que ocorram diversas mudanças manifestadas ou não por sinais e sintomas que caracterizam a síndrome climatérica, trazendo modificações transitórias e permanentes com desconfortos, alterações físicas e emocionais que influenciam a saúde das mulheres (Selbac et al. 2018). As milhares de mulheres nesta fase, principalmente as ribeirinhas, necessitam de acesso à saúde, 
sendo esse um grande desafio, que implica em atenção das políticas públicas e programas para garantia dos preceitos da Universalidade, Equidade e Integralidade, pilares do SUS (Gallon \& Wender, 2012).

No Brasil, as informações epidemiológicas sobre as populações ribeirinhas da Amazônia, àquelas que vivem às margens de rios e lagos na Amazônia brasileira, são escassas. O isolamento devido ao relevo e dimensões geográficas colossais da Amazônia imprimem inúmeras restrições, onde, quase sempre, os ribeirinhos são povos privados de saneamento básico, em que o esgoto é lançado a céu aberto, sem acesso a água tratada, energia elétrica e serviços de saúde; situações estas que estão entre os mais importantes fatores sociais determinantes do processo saúde-doença (Gama et al. 2018 \& Reis et al. 2020).

A invisibilidade, a dispersão física, o isolamento e os problemas socioeconômicos aliados à ausência ou à insuficiência de políticas públicas de saúde e de saneamento e a escassez de recursos direcionados a essas comunidades, têm contribuído para alicerçar este grave cenário de carências (Brasil, 2013). A carência multidimensional dos ribeirinhos amazônicos, constitui-se como determinante para enfrentamento das dificuldades impostas, os impelindo a lançar mão de sua cultura e de conhecimentos milenares a respeito de si mesmo e da floresta, nos processos de autocuidado e de cura.

Sendo assim, o objetivo deste trabalho foi compreender as práticas e saberes das mulheres ribeirinhas relativas ao período de climatério e do autocuidado. Neste contexto, o estudo poderá contribuir para a construção e efetividade de políticas públicas para essa população, para diminuição das iniquidades e favorecendo a qualidade de vida das mesmas.

\section{Metodologia}

Estudo descritivo exploratório, com abordagem qualitativa, realizado em comunidades ribeirinhas situadas ao longo da margem esquerda do Rio Amazonas. Estas comunidades eram assistidas quinzenalmente pela equipe de saúde fluvial.

Os dados foram coletados através de entrevistas semiestruturadas, individuais, cujas falas foram gravadas para manter a fidedignidade das informações. O instrumento de coleta continha dados sociodemográficos e questões norteadoras, sendo elas: a) A senhora sabe o que é climatério ou menopausa? A senhora pode falar um pouquinho sobre como foi/está sendo sua experiência nessa fase? b) O que a senhora sente nessa fase? Percebeu alguma mudança no seu corpo, nos seus sentimentos ou relações familiares? c) O que a senhora usava ou fazia para se cuidar ao sentir essas mudanças? d) Existe na comunidade alguma pessoa que ajuda as mulheres nessa fase? Que tipo de ajuda ela oferece?

As participantes da pesquisa foram identificadas no momento do acolhimento, enquanto aguardavam atendimento na unidade fluvial e selecionadas a partir dos critérios de inclusão e exclusão. Participaram mulheres na faixa etária entre 40 e 65 anos de idade, residentes nas comunidades ribeirinhas da margem esquerda do rio Amazonas. Foram excluídas desse estudo, mulheres indígenas, com alteração na saúde mental ou que apresentaram alguma doença/situação que impossibilitou a realização da entrevista.

A técnica optada para definição do tamanho amostral foi a da saturação teórica, porém todas as mulheres dentro dos critérios que vieram para o atendimento foram recrutadas e entrevistadas (Nascimento et al. 2018).

A análise das informações e interpretação dos dados foi baseada na técnica de análise temática de Leininger e McFarland (2002). Cada participante recebeu uma identificação em letras e números para garantir o sigilo dos dados (E1, E2 ...E24).

Na construção deste estudo, observou-se as normas legais preconizadas na Resolução 466/2012. Autorizado pela Gerência de Gestão de Educação na Saúde (GESAU) da Secretaria Municipal de Saúde de Manaus (Autorização número 12/2016). Aprovado pelo Comitê de Ética em Pesquisa da Universidade Federal do Amazonas (Protocolo: CAAE: 53773416.8.0000.5020). Foi apresentado um Termo de Consentimento Livre e Esclarecido que foi assinado por todas as participantes da pesquisa. 


\section{Resultados}

Vinte e quatro ribeirinhas participaram do estudo. Vinte $(83,2 \%)$ dessas mulheres viviam com o companheiro, uma $(4,2 \%)$ estava divorciada, uma $(4,2 \%)$ solteira e duas $(8,4)$ eram viúvas. Quanto à escolaridade, cinco $(20,8 \%)$ eram alfabetizadas, seis $(25 \%)$ possuíam o ensino fundamental incompleto, seis $(25 \%)$ completaram o ensino fundamental, cinco (20,8\%) finalizaram o ensino médio e duas (8,4\%) possuíam o ensino superior. Quanto à ocupação, nove (37,5\%) das participantes declararam ser do lar, três (12,5\%) agricultoras, quatro (16,8\%) autônomas (vendedoras), uma (4,2\%) trabalhava em serviços gerais, duas $(8,4 \%)$ professoras, uma (4,2\%) desempregada e quatro $(16,8 \%)$ aposentadas.

As falas das mulheres ribeirinhas possibilitaram a construção de três categorias temáticas: a percepção do climatério/menopausa, práticas de autocuidado no climatério e sistemas de cuidado em saúde no climatério. A descrição dos sinais, sintomas e experiência pessoal, de uma forma geral, foram agrupadas na primeira categoria. Já a segunda, abrange todas as práticas que as mulheres ribeirinhas realizam para que pudessem ter uma experiência de melhor qualidade de vida no climatério. A terceira categoria apresenta o sistema de atenção utilizado quando as mulheres percebem as mudanças físicas e emocionais relativas a esse momento.

\subsection{Mulheres Ribeirinhas: Percebendo-se e experenciando o climatério/menopausa}

A pesquisa revelou que as mulheres ribeirinhas desconheciam a palavra climatério, contudo tinham maior familiaridade com a terminologia menopausa, além de apreender que nesse momento está presente alterações físicas e emocionais. Suas experiencias revelam que esse período era marcado por sentimentos e vivências negativas, percepção de sinais e sintomas físicos e psíquicos, como se pode apreender nas seguintes falas:

"[...] era o fim de sangue, tipo uma fraqueza”. (E4).

"Eu não sei explicar, só sei que a mulher para de menstruar'. (E15)

“A gente começa a sentir tontura, dores na pente (região supra púbica), aí já é o início da menopausa. Já me dá tonteira quando eu menstruo, passa muitas horas com dor de cólica. Antes de eu menstruar eu passo quase que uns quinze dias com enjoo para poder menstruar, e depois que menstruar passa”. (E10)

Outra mulher, relatou o conceito de menopausa como sendo a soma da sintomatologia do climatério com o marco da última menstruação.

“[...] é um ciclo da mulher quando ela para de menstruar e começa a sentir aquele calor, vagina ressecada... ela tá entrando na menopausa”... (E21)

É importante destacar que as mulheres ribeirinhas percebem as mudanças de ordem tanto físico-biológicas quanto psíquicas durante o climatério:

"Sinto uns quebrantamentos (dores) no corpo, sente falta de ar, tontura, dor de cabeça". (E16)

“Eu sentia muitas dores nas minhas pernas”. (E24)

“Muitas dores eu sinto na coluna”. (E23)

“Falta de disposição de fazer o trabalho”. (E24)

“Me dava um tremor de gelar o sangue todinho". (E3) 
"Aquele calafrio, sabe, uma hora é quentura, quentura, outra hora está frio. Sempre dá essas coisas, aí desejos também, vontade de comer as coisas tudo como se tivesse grávida. Dor nos ossos, eu sinto muita dor nas juntas" (E22) "Abaixava a pressão e ficava tendo mal-estar na escola, um gesto assim meio irritante" (E11)

“[...] tem aquele fogacho, né, que chama. Quentura, aquele mal-estar que as mulheres sentem. Outras a gente não sabe nem explicar que é algo assim muito desconfortável... algumas vem com hemorragia, sangramento abundante, o fluxo de sangue se torna muito, muito, muito mesmo. E, deixa eu ver...traz um pouco de calcificação óssea também" ( E20)

Observa-se, em sequência, o grupo de manifestações psíquicas relatadas pelas ribeirinhas:

"Eu me sentia ruim (triste), ai naqueles primeiros dias eu passava quase assim acamada" (E5)

"A pessoa fica histérica, com disimpaciência (impaciência), dor de cabeça, mal-estar, estresse, essas coisas". (E6)

"Eu fico triste, dá vontade de chorar" (E21)

"Se irritar por qualquer coisa, do nada as vezes dá aquela irritação"... (E22)

"[...] eu tenho muito sono". (E23)

“[...] eu fiquei mais aborrecida, agressiva, mas eu consigo controlar”... (E11)

Houve, também, casos de relatos em que se observou que algumas mulheres ribeirinhas experenciaram a fase do climatério sem queixas, como nos depoimentos:

"[...] eu parei de menstruar e não senti nada, nada, nada. Minhas amigas contaram que tiveram muita dor de cabeça, quentura, é isso ... em mim não deu nada". (E17)

"Eu não tive esses negócios não, mas tem muita gente que diz que é muito ruim, dá dor de cabeça, dá sistema nervoso, dá muito calor aí outra hora dá muito frio e eu sei que eu não tive essas coisas, não, graças a Deus”. (E12)

\subsection{Mulheres Ribeirinhas e as vivencias de autocuidado}

As mulheres ribeirinhas vivenciam a necessidade de se autocuidar com vista a minimizar os sinais e sintomas desse período, para a promoção da saúde, do bem-estar e da qualidade de vida. Diante disso, verifica-se as vivências do autocuidado das mulheres ribeirinhas no uso de plantas medicinais, em que as entrevistadas referem a utilização de ervas para amenizar a sintomatologia do período, conforme a Quadro 1. 
Quadro 1 - Relação das plantas medicinais e dos depoimentos das mulheres ribeirinhas.

\begin{tabular}{|c|c|c|}
\hline \multicolumn{2}{|c|}{ Plantas Medicinais } & \multirow{2}{*}{$\begin{array}{l}\text { Depoimento das Mulheres Ribeirinhas (modo de usar e } \\
\text { indicação) }\end{array}$} \\
\hline Nome Popular & Nome Científico & \\
\hline Arruda & Ruta graveolens & $\begin{array}{l}\text { "Bate elas juntas, a casca de jucá e a folha da arruda; faz o chá e toma } \\
\text { toda vez que sente os sintomas que diminui a sensação ruim". (E1) }\end{array}$ \\
\hline Jucá & Libidibia férrea & $\begin{array}{l}\text { "Tomava } 3 \text { vezes ao dia, de manhã, meio dia e à noite em forma de } \\
\text { chá". (E6). } \\
\text { "Em casa a gente toma é direto, é bom pra tudo, para inflamação e } \\
\text { problemas do útero". (E10) }\end{array}$ \\
\hline Pimenta-do-reino & Piper nigrum & $\begin{array}{l}\text { "Chá eu tomava toda vez que eu sentia alguma coisa. Aí ela (a } \\
\text { menstruação) ficava normal e nunca ficou exagerada como vem na } \\
\text { minha filha". (E4) }\end{array}$ \\
\hline Uxi-amarelo & Endopleura uchi & $\begin{array}{l}\text { "Chá da casca pra amenizar a menstruação que estava muito, sabe. } \\
\text { Eu não tomei sempre, tomei duas vezes e melhorei..."(E16) }\end{array}$ \\
\hline Mutuquinha & Justicia pectoralis & \multirow{2}{*}{$\begin{array}{l}\text { "O chá dos três mutuquinha, mangarataia e pimenta do reino. Toma } \\
\text { em toda a menstruação, uma vez no primeiro dia até parar." (E5) }\end{array}$} \\
\hline Mangarataia & Zingiber officinale & \\
\hline Sara-tudo & Justicia calycina & \multirow{4}{*}{$\begin{array}{l}\text { "Tomava muito chá e se lavava. A gente toma chá de... Sara-tudo, } \\
\text { unha-de-gato e pobre-velho... algodão-roxo, tudo é bom, a gente usa } \\
\text { muito aqui no interior." (E14). "Tomo para não dar inflamação." } \\
\text { (E11) }\end{array}$} \\
\hline Unha-de-gato & Uncaria tomentosa & \\
\hline Pobre-velho & Costus & \\
\hline Algodão-roxo & Gossypium & \\
\hline Paracanaúba & Aspidospermum sp. & \multirow{3}{*}{$\begin{array}{l}\text { "Chá de paracanaúba, castanheira e casca de crajirú é o que tomam } \\
\text { aqui na região para inflamação." (E18) }\end{array}$} \\
\hline Castanheira & Bertholletia excelsa & \\
\hline Crajirú & Arrabidaea chica & \\
\hline Agrião & Nasturtium officinale & \multirow{3}{*}{$\begin{array}{l}\text { "Chá de agrião controla a pressão e normaliza quando vinha muito a } \\
\text { minha menstruação" (E11). } \\
\text { "Chá de cidreira e camomila ajuda a acalmar". (E22) }\end{array}$} \\
\hline Cidreira & Lippia alba & \\
\hline Camomila & Matricaria chamomilla & \\
\hline Amora & Morus & "Já mandaram eu tomar chá da folha de amora." (E10) \\
\hline
\end{tabular}

Fonte: Banco de dados dos autores.

As participantes, ainda relataram a importância do autocuidado, da alimentação e a atividade física com ênfase em manter os hábitos saudáveis no seu dia-dia:

“[...] comer feijão, muita carne de soja, muito grão de bico...você precisa se alimentar, porque isso vai fazer bem na menopausa...comer morango, bastante morango porque ele ajuda nos ossos, ajuda nas vitaminas necessárias, que ele tem vitamina $D ”$. (E20)

“Para ajudar a acalmar.. o suco do maracujá é muito bom”. (E22)

“Já tem muito tempo que eu faço caminhada, eu me exercito... e eu me sinto melhor”. (E23)

Uma das mulheres ribeirinhas referiu a importância da assistência médica durante esse período para o autocuidado:

"Simplesmente o que aconselho é acompanhamento médico, medir o hormônio. Sempre eles aconselham dessa forma”. (E21)

Ainda foi exposto a prática da automedicação das participantes, onde elas relatam o uso de medicamento quando apresentam sintomas da síndrome climatérica:

“... eu tomo aquele remédio da saúde da mulher, ele é próprio para menopausa, está escrito no vidro: regulador menstrual”. (E10) 
“Quando eu estou sentindo dor de cabeça, ai eu tomo uma dipirona... paracetamol...” (E22)

Como observado, as mulheres ribeirinhas ao experienciarem as mudanças climatéricas realizam as seguintes práticas de autocuidado: com a utilização de plantas medicinais na forma de chá, a ingesta de alimentos que sejam fonte de nutrientes como a vitamina D, a realização de atividades físicas, a procura pelo auxílio médico e automedicação. Essas práticas são reflexo de conhecimentos que elas adquirem da rede de suporte que possuem.

\subsection{Mulheres ribeirinhas experienciando os sistemas de cuidado em saúde}

As mulheres revelaram que o sistema de cuidado em saúde é proveniente do cuidado familiar-popular e do cuidado profissional. Com isso, apreende-se com as mulheres ribeirinhas que o saber é propagado de geração em geração, num processo de transgeracionalidade, revelando uma comunidade ativa no processo de apreensão, transmissão e construção do saber. Apresenta a família com um importante papel na apropriação e transmissão de conhecimento entre as gerações. Destacase também o valor da interrelação mútua e recíproca nas trocas de experiências, de informações e de aprendizado, com elementos da educação popular, como referenciado pelo educador Paulo Freire (Freire, 2020).

As mulheres Ribeirinhas, relataram que suas fontes de apoio eram àqueles à sua volta: família, vizinhas, amigas e religiosas, enfim pessoas da comunidade. A Figura 1 apresenta o Sistema Familiar-popular de Cuidado das mulheres ribeirinhas.

Figura 1 - Sistema Familiar-popular de cuidado das mulheres ribeirinhas.

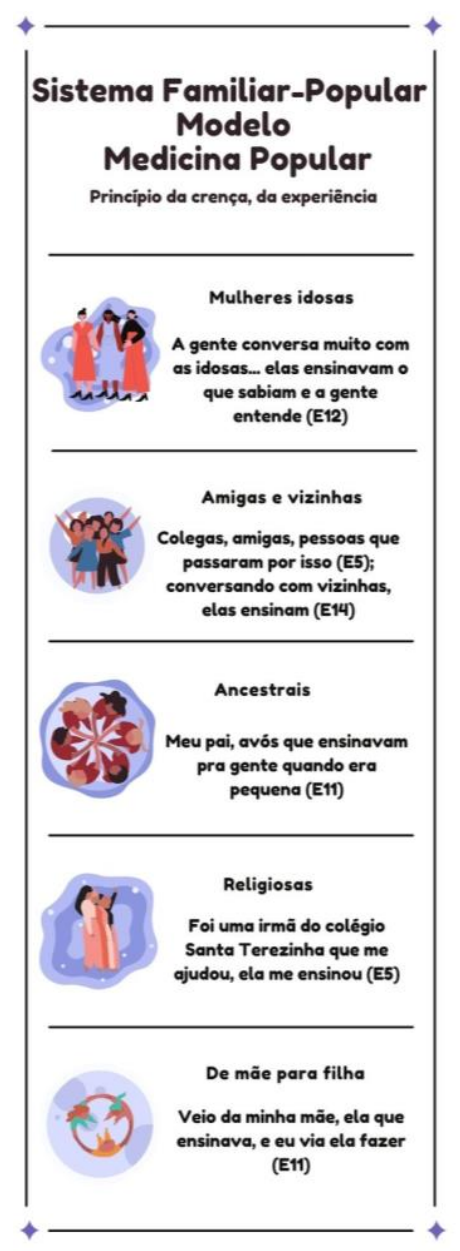

Fonte: Banco de dados dos autores. 
Nesse contexto, foi relevado que as mulheres ribeirinhas são cuidadas primeiramente pelo agente Comunitário de Saúde e posteriormente a cada 15 dias, pela equipe de saúde composta principalmente pelos enfermeiros, médicos, psicólogos e dentistas que vão até a comunidade, visualizado como uma espécie de sistema de cuidado profissional. Caso não haja resolutividade, essas mulheres são encaminhadas para a assistência fora da comunidade através das unidades fluviais de saúde. Sendo assim, as falas revelavam a busca pela assistência profissional como observa-se nas seguintes falas da Figura 2:

Figura 2 - Sistema profissional de cuidado da mulher ribeirinha.

\section{SISTEMA PROFISSIONAL \\ DE CUIDADO \\ (MODELO BIOMEDICO)}

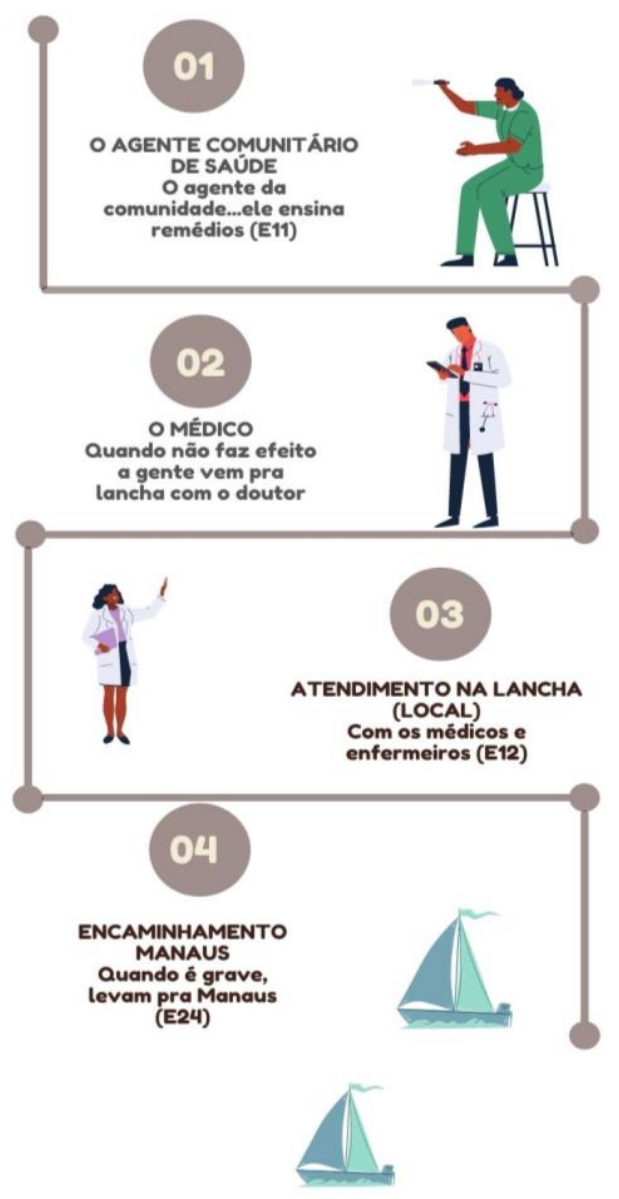

Fonte: Banco de dados dos autores.

\section{Discussão}

O saber da mulher ribeirinha sobre o climatério/menopausa é multidimensional, heterogêneo, culturalmente construído e influenciado pelo acesso a informações e nível educacional de cada uma. Neste estudo, todas eram alfabetizadas porém das 24 participantes, dezessete $(70,8 \%$ ) possuíam baixa escolaridade com níveis de formação entre, apenas alfabetizadas e ensino fundamental. Esse fato condiciona a possibilidade de dificuldades no acesso à informação, observação, compreensão $\mathrm{e}$ interpretação mais acurada, podendo limitar a capacidade de autocompreensão, autocuidado e busca de assistência de terceiros, 
quando necessário. A baixa escolaridade pode influenciar negativamente no modo pelo qual as ribeirinhas gerenciam sua saúde assim como a implementação de ações que levem em consideração a realidade local (Guimarães, 2020).

A percepção do Climatério/Menopausa para essas mulheres traduz a interpretação de acontecimentos experienciados no dia a dia, as crenças e mitos formados, determinando de que forma as mulheres irão vivenciar essa fase. Logo, a forma que enfrentam os sintomas e as alterações físicas e psicológicas recebem influência do meio social, cultural e econômico no qual cada mulher está inserida, fato descrito por Alves et al. (2020). Foi observado que nas falas há uma visão negativa do climatério, na qual há uma ênfase nos sintomas desconfortáveis. De acordo com Leite et al. (2020) e Von Rondon et al. (2020) a falta de informações a respeito do climatério, faz com que as mulheres associem esse período a algo negativo, com associação de pensamentos negativos acerca da própria imagem e com implicações nos desconfortos físicos. Para Pinto et al. (2021) essa lógica de percepção feminina tão negativa sobre o climatério é devida as profundas fragilidades que informação que devem ser dispensadas por processos educativos dialógicos e que tragam a mulher em um contexto integral e social.

Possivelmente essa percepção foi historicamente construída por influência da mídia, indústria farmacêutica e até mesmo dos profissionais de saúde por identificarem o climatério com um evento patológico, não natural e basear suas práticas no processo de curar e não de cuidar conforme pode-se observar no desenrolar literário sobre a temática (Brasil, 2008).

Para as mulheres ribeirinhas, desse estudo, a experiência vivenciada na síndrome climatérica está fortemente relacionada à percepção dos sinais e sintomas. Climatério e Menopausa para elas são sinônimos e apresentam a manifestação de sintomas físico-biológicos e psíquicos, como dores no corpo, mal-estar, enjoo, falta de ar, vertigem, ondas de calor, calafrio, ressecamento vaginal, irregularidades na duração dos ciclos menstruais e na quantidade do fluxo menstrual, problemas ósseos, hipotensão, desânimo em realizar atividades do dia-a-dia, irritabilidade, labilidade emocional, diminuição da libido, sonolência ou insônia.

O climatério não é um período pontual na vida da mulher, compreende o processo de envelhecimento feminino e as alterações na imagem do corpo. Por isso, essa fase muitas vezes remete à significação negativa pois anuncia a chegada da velhice. As mulheres de meia idade possuem percepção mais negativa sobre a experiência de envelhecer, associando-o a perda da autonomia, o declínio da saúde e a decadência (Carrara et al., 2020). Nesse estudo a maioria das mulheres vivia com companheiros e era do lar, Silva, Rocha e Caldeira (2018) descreve que mulheres com companheiro e sem trabalho formal são mais propensas a apresentar percepção negativa da saúde.

Buscando, então, o alívio dos sintomas da síndrome climatérica, as mulheres ribeirinhas fizeram o uso de 17 tipos de plantas de uso medicinal. A utilização de plantas medicinais é de suma relevância, sendo uma importante alternativa, principalmente em locais onde o acesso a assistência à saúde é difícil ou precário, como na maioria das comunidades ribeirinhas. A fitoterapia na vida da mulher ribeirinha tem função fundamental no fortalecimento da prática de cuidados femininos (Valeriano et al., 2019). A acessibilidade e variedade de plantas disponíveis na comunidade podem auxiliar desde os incômodos com a menstruação até alterações hormonais do organismo como também em outros desconfortos gerais (Oliveira, 2016). A utilização das plantas medicinais também valoriza o papel da mulher na comunidade uma vez que elas cultivam e repassam o conhecimento adquirido, fortalecendo a cultura e o saber através da transgeracionalidade, o que é retratado no presente estudo. Ainda neste contexto o estudo de Souza et al. (2020) demostrou e efetividade de uma variedade de fitoterápicos para a redução dos sintomas vasomotores, neuropsíquicos, disfunção sexual, alterações urogenitais e distúrbios metabólicos, contribuindo assim, para uma diminuição das queixas climatéricas.

A atividade física e alimentação saudável foram práticas de autocuidado realizadas por elas, são recursos terapêuticos valiosos que devem ter seu uso estimulado. A promoção da alimentação saudável e a manutenção do peso adequado também são essenciais na manutenção das funções orgânicas em boas condições. O cuidado nutricional das mulheres no climatério 
deve ser intensificado através de uma alimentação balanceada, voltada para a oferta dos componentes essenciais diários (Brasil, 2008).

Foi verificado o uso da automedicação. A dinâmica de vida e condições sócio econômicas, culturais e geográficas dos ribeirinhos, contribui para o entendimento que a prática de automedicação, como estratégia de autocuidado e até de sobrevivência, é possível em uma realidade de vida em que há inúmeras dificuldades e limitações como a ausência de controle governamental, profissionais não autorizados prescrevendo medicamentos, facilidade na comercialização sem prescrição médica, dificuldade de acesso a serviços de saúde e a profissionais qualificados (Gama \& Secori, 2020). Apesar dessa realidade a automedicação deve ser considerada uma prática danosa, pois pode levar a tratamentos pouco seguros e ineficazes. A realidade vivenciada pelas ribeirinhas, neste estudo, apresenta uma rede de cuidado e assistência em modelos utilizados harmoniosamente: Sistema Familiar- Popular de Cuidado (Modelo Medicina popular) e Sistema Profissional de Cuidado (Modelo Biomédico).

O Sistema Familiar Popular de Cuidado chamado por Cruz (2020) de Modelo de Medicina Popular parte do princípio da crença, da experiência, do notório saber e cuidados e caracteriza-se por evolucionismo histórico que agregou práticas aos saberes ao longo da história natural. Em algumas regiões do Brasil, como nas comunidades ribeirinhas, o remédio caseiro, os chás, rezas e crendices são, muitas vezes, os únicos tratamentos disponíveis corroborando com a importância do Sistema no processo de cuidado, saúde e doença. Nesse estudo foi verificado que mesmo havendo a presença quinzenal da Unidade Básica de Saúde Fluvial, o autocuidado baseado no conhecimento adquirido ao longo dos anos e das experiências vividas na comunidade é fortemente difundido e utilizado. É possível verificar que o fato de nenhuma participante do estudo conhecer o termo científico climatério, não usual na comunidade, justifica-se por sua fonte primária de informações ser o Sistema Familiar- Popular de Cuidado.

Deve-se considerar a influência que os fatores exercem na vida de cada mulher, pois um cuidado culturalmente coerente aproxima o saber popular do profissional. Os resultados apontam que por mais que as mulheres ribeirinhas valorizem o saber biomédico, ao vivenciar as mudanças da síndrome climatérica, a grande maioria delas optam, inicialmente, pelo saber transgeracional e popular adquirido tanto no seio familiar, quanto na comunidade em que residem.

\section{Considerações Finais}

As mulheres ribeirinhas desse estudo, possuem um saber significativo sobre a síndrome climatérica e a identificam de acordo com suas percepções, vivências e experiências construídas através dos simbolismos culturais e principalmente através da percepção dos sinais e sintomas. Realizam práticas de autocuidado (como o uso de plantas medicinais, automedicação, nutrição e atividades físicas), adquiridas através de seu sistema de cuidado de saúde Familiar-Popular (Modelo Medicina Popular) que preserva costumes e crenças e utilizam o Sistema Profissional de Cuidados (Modelo Biomédico), demonstrando que é possível a complementaridade entre modelos, na construção de um sistema de saúde forte, para assistir e contribuir com uma saúde de melhor qualidade.

Permite reflexões sobre o desafio que é a promoção e assistência à saúde de povos longínquos. Instiga os profissionais a contemplar a transculturalidade nos processos de trabalho para uma atenção integral de qualidade permitindo assim novas perspectivas assistenciais a fim de promover melhor qualidade de vida à mulher ribeirinha.

Portanto, verifica-se o quanto ainda pode ser difícil realizar pesquisas em sujeitos moradores de áreas ribeirinhas da região amazônica, levando em consideração as distâncias percorridas, a logística de acesso as comunidades e a autorização dos próprios participantes em colaborar com estudos dessa natureza. Nessa situação, ocorrem algumas resistências por parte dos sujeitos em expor assuntos de cunho pessoal, impossibilitando a coleta de mais dados subjetivos. 
Assim, sugerem-se estudos que possam verificar as propriedades das plantas medicinais utilizadas por essas mulheres, de maneira a complementar o conhecimento tradicional já posto, bem como, agregar metodologias da Assistência Farmacêutica na propositura destes tratamentos medicinais. Por fim, é importante a produção, validação e incorporação de tecnologias cuidativo-educacionais para que os conhecimentos precisos possam chegar junto a esta população, levando em consideração as particularidades de cada indivíduo.

\section{Referências}

Alves, E. R. P., Nóbrega, A. A. M., Rodrigues, A. V. de F., \& Santos, R. M. dos. (2020). Climatério: perspectivas de mulheres profissionais da saúde do hospital universitário Nova Esperança, João Pessoa-PB. Revista de Ciências da Saúde Nova Esperança. 18(2): 61-72. https://doi.org/10.17695/revcsnevol18n2p61-72.

Brasil. Ministério da Saúde (2008). Manual de Atenção à Mulher no Climatério/Menopausa http://bvsms.saude.gov.br/bvs/publicacoes/manual_atencao_mulher_climaterio.pdf.

Brasil. Ministério da Saúde (2013). Política Nacional de Saúde Integral das Populações do Campo e da Floresta. https://bvsms.saude.gov.br/bvs/publicacoes/politica_nacional_saude_populacoes_campo.pdf

Carrara, F. F., Vinagre, C. G. C. de M., \& Pereira, L. L. (2020). Percepção do envelhecimento: mulheres de meia idade e idosas que buscam por procedimentos estéticos. Id on Line Revista Multidisciplinar e de Psicologia. 14(49): 38-50. doi: 10.14295/idonline.v14i49.2309

Cruz, R. (2020). Saberes e estratégias de cuidado na medicina popular - uma revisão epidemiológica em medicina popular ou fitoterapia. Revista Cathedral, 2(3), 157-169. Recuperado de http://cathedral.ojs.galoa.com.br/index.php/cathedral/article/view/201

Freire, P. Pedagogia da autonomia: saberes necessários à prática educativa. (2020). Paz e terra.

Gallon, C. W., \& Wender, M. C. O. (2012). Estado nutricional e qualidade de vida da mulher climatérica. Revista Brasileira de Ginecologia e Obstetrícia, 34(4), 175-183. Doi.org/10.1590/S0100-72032012000400007.

Gama, A. S. Muri., Secoli, S. R. (2020). Práticas de automedicação em comunidades ribeirinhas na Amazônia brasileira. Revista Brasileira de Enfermagem 73(5):e20190432 https://doi.org/10.1590/0034-7167-2019-0432.

Gama, A.S.M., Fernandes, T.G., Parente, R. C. P., \& Secoli, S. R. (2018). Inquérito de saúde em comunidades ribeirinhas do Amazonas, Brasil. Cadernos de Saúde Pública. 34(2):e00002817. doi: 10.1590/0102-311X00002817.

Guimarães, A. F., Barbosa, V. L. M., Silva, M. P. da., Portugal, J. K. A., Reis, M. H. da S., \& Gama, A. S. M. (2020). Acesso a serviços de saúde por ribeirinhos de um município no interior do estado do Amazonas, Brasil. Revista Pan-Amazonica de Saude.11:e202000178. 10.5123/S2176-6223202000178.

Instituto Brasileiro de Geografia e Estatística (2019). https//educa.ibge.gov.br/jovens/conheca-o-brasil/população/18320-quantidade-de-homens-emulheres.html.

Leite, T. A. S., Nunes, J. S. S., Pereira, A. de J., \& Silva, M. L. .(2020). Conhecimento de mulheres jovens sobre a menopausa e sintomas climatéricos. Brazilian Journal of health Review. 3(3) 7204-7212. doi:10.34119/bjhrv3n3-249.

Madeleine, L. McFarland, M. R. (2002). Transcultural nursing: concepts, theories, research \& practice. Mac Graw-Hill.

Nascimento, L. de C. N., Souza, T. V. de., Oliveira, I. C. dos S., Moraes, J. R. M. M. de., Aguiar, R. C. B. de., \& Silva, L. F. da. (2018). Saturação teórica em pesquisa qualitativa: relato de experiência na entrevista com escolares. Revista Brasileira de Enfermagem. 71(1):243-8. https://doi.org/10.1590/0034-71672016-0616.

Oliveira, A. P. (2016). O conhecimento tradicional sobre plantas medicinais no âmbito da saúde da mulher: uma perspectiva no contexto do produto tradicional fitoterápico. Revista Fitos, Supl, 1-62, doi: 10.5935/2446-4775.20160043

Reis, M. H. da S., Portugal, J. K. A., Mariño, J. M., Barros, W. da S., Dantas, J. de S., Souza, T. T. G. de., Reis, Y. da S. dos., Germano, S. N. F., Barão, É.J.da S., \& Freitas, D. L. A. de. (2020). O impacto do advento de uma Unidade Básica de Saúde Fluvial na assistência aos povos ribeirinhos do Amazonas. Revista Eletrônica Acervo Saúde. 53 (Sup)1-6. https://doi.org/10.25248/reas.e3631.2020.

Selbac, M. T., Fernandes, C. G. C., Marrone, L. C. P., Veira, A. G., Silveira, E. F. da, \& Martins, M. I. M. (2018). Mudanças comportamentais e fisiológicas determinadas pelo ciclo biológico feminino - climatério à menopausa. Aletheia. 51(1-2): 177-190. http://pepsic.bvsalud.org/pdf/aletheia/v51n1-2/v51n12a16.pdf.

Silva, V. H., Rocha, J. S. B., \& Caldeira, A. P. (2018). Fatores associados à autopercepção negativa de saúde em mulheres climatéricas. Ciência \& Saúde Coletiva. 23(5):1611-1620. https://doi.org/10.1590/1413-81232018235.17112016.

Souza, A. S., Ribeiro Junior, O. C., Ribeiro, J. S. S., Mendonça, L. B., Melo, J. M. R. de, \& Araújo, T. S. de. (2020). A utilização de fitoterápicos no manejo de mulheres no climatério/menopausa. Research, Society and Development, 9(9), e415997416. https://doi.org/10.33448/rsd-v9i9.7416

Pinto, V. L., Wanderley, M. C. A., \& Neto, J. M. W. D. (2021). Vivendo o Climatério: Percepção de Mulheres Usuárias de Unidade de Saúde da Família em Recife-PE. Pesquisa, Sociedade e Desenvolvimento, 10 (16), e375101623892-e375101623892. https://doi.org/10.33448/rsd-v11i2.23574 
Research, Society and Development, v. 11, n. 3, e17511326391 2022

(CC BY 4.0) | ISSN 2525-3409 | DOI: http://dx.doi.org/10.33448/rsd-v11i3.26391

Valeriano, F. R., Savani, F. R., \& Silva, M. R. Valeriano da .(2019). O uso de plantas medicinais e o interesse pelo cultivo comunitário por moradores do bairro São Francisco, município de Pitangui, MG. Interações (Campo Grande). 20 (3):891-905. https://doi.org/10.20435/inter.v0i0.1846.

Von Rondon, K. W. S., de Lima Pimentel, A. C., Toledo, G. S., \& da Silva, L. M. M. (2020). Percepções das mulheres no climatério, um novo ciclo de vida? Revista de APS (Supl. 2): 287-296. 